\title{
Atomic Force Microscope
}

National Cancer Institute

\section{Source}

National Cancer Institute. Atomic Force Microscope. NCI Thesaurus. Code C78804.

A scanning probe microscope that has nanometer resolution. It consists of a microscale cantilever, a laser, an array of photodiodes and a detection system. 